\title{
AVALIAÇÃO DE RESISTÊNCIA EM CULTIVARES DE ARROZ AO ATAQUE DO PERCEVEJO-DO-COLMO, TIBRACA LIMBATIVENTRIS STAL, 1860 (HEMIPTERA: PENTATOMIDAE)
}

\author{
J.R. Souza ${ }^{1 *}$, E. Ferreira ${ }^{2 \dagger}$, A.L. Boiça Junior ${ }^{3}$, A. Cargnelutti Filho ${ }^{4 * *}$, E.F. Chagas ${ }^{5}$, J.M. Mondego ${ }^{6}$ \\ ${ }^{1}$ Universidade Estadual Paulista, Faculdade de Ciências Agrárias e Veterinárias, Departamento de Ciências \\ Exatas, Via de Acesso Prof. Paulo Donato Castellane, s/no, CEP 14849-900, Jaboticabal, SP, Brasil. E-mail: \\ joseaneagro@yahoo.com.br
}

\section{RESUMO}

A resistência de cultivares de arrozao ataque do percevejo-do-colmo, Tibraca limbativentris Stal, 1860 (Hemiptera: Pentatomidae), foi avaliada em experimento conduzido em casa-de-vegetação na Embrapa Arroz e Feijão no período de dezembro de 2004 a fevereiro de 2005, em delineamento experimental de blocos aumentados de Federer com dez repetições. Na avaliação foram considerados sete caracteres de resistência ao ataque do inseto. Foram utilizados 64 cultivares de arroz, sendo 60 considerados tradicionais e quatro cultivares mais atuais como testemunhas: Cica 8, Bonança, Primavera e BR IRGA 409. Os resultados obtidos apontaram que os cultivares Nenenzinho, Miúdo Branco, Lageado Ligeiro, Guabirú, Branco Tardão, Agulhinha do Seco, Arroz do Governo, Arroz Misturado, Vermelho Trinca Ferro, Vermelhão, Chatão, Cutião, Vermelho, Bacaba Branco, Catetão, Buriti, Bacaba, Agulha, Arroz Comum, Vermelho, Pingo D’ Água, Marabá Branco, Come Cru Vermelho e Agulhão destacaram-se como resistentes possivelmente do tipo antibiose, enquanto os cultivares Pela Mão, Arroz do Governo, Cana Roxa, Come Cru Branco, Bico Preto, Pingo de Ouro, Matão, Gojobinho, Buriti, Rabo de Burro, Poupa Preguiça, Vermelho Trinca Ferro, Miúdo Branco, Ligeiro Curto, Vermelho Agulha, Agulhão, Marabá Branco, Gojoba $\backslash 75$ Dias $\backslash$ Ligeiro, Pingo D'Água, Arroz Comum e Vermelho mostraram-se provavelmente como tolerantes ao ataque do percevejo-do-colmo podendo, portanto, ser devidamente explorados em programas de melhoramento para resistência a T. limbativentris.

PALAVRAS-CHAVE: Oryza sativa, melhoramento de plantas, resistência de plantas a insetos, tipos de resistência.

\section{ABSTRACT}

REVALUATIONOF RESISTANCEOF RICECULTIVARSTOTHEATTACKOFTHESTEMBUG TIBRACA LIMBATIVENTRIS STAL, 1860 (HEMIPTERO: PENTATOMIDAE). Rice cultivars were evaluated in regard to their resistance to attack by the stem bug Tibraca limbativentris Stal, 1860 (Hemiptera: Pentatomidae) in an experiment carried out in the greenhouse at Embrapa Arroz and Feijão, Brazil, in randomized augmented Federer blocks with ten replications. Seven traits related to plant resistance were evaluated. A total of 64 rice cultivars were used, 60 of which were considered traditional, and 4 of which were more recent cultivars, used as controls: Cica 8, Bonança, Primavera and BR IRGA 409. The results showed that the cultivars Nenenzinho, Miúdo Branco, Lageado Ligeiro, Guabirú, Branco Tardão, Agulhinha do Seco, Arroz do Governo, Arroz Misturado, Vermelho Trinca Ferro, Vermelhão, Chatão, Cutião Vermelho, Bacaba Branco, Catetão, Buriti, Bacaba, Agulha, Arroz Comum, Vermelho, Pingo D’Água, Marabá Branco, Come Cru

\footnotetext{
${ }^{2}$ Embrapa Arroz e Feijão, Centro Nacional de Pesquisa de Arroz e Feijão, Santo Antônio de Goiás, GO, Brasil.

${ }^{3}$ Universidade Estadual Paulista, Faculdade de Ciências Agrárias e Veterinárias, Departamento de Fitossanidade, Jaboticabal, SP, Brasil.

${ }^{4}$ Universidade Federal de Santa Maria, Departamento de Fitotecnia, Centro de Ciências Rurais, Santa Maria, RS, Brasil.

${ }^{5}$ Universidade Estadual do Maranhão, Departamento de Fitotecnia e Fitossanidade, São Luís, MA, Brasil.

${ }^{6}$ Engenheira Agrônoma, São Luis, MA, Brasil.

*Mestranda em Agronomia - Genética e Melhoramento de Plantas.

**Bolsista PQ CNPq.

In memorian.
} 
Vermelho and Agulhão stood out as resistant, possibly of the antibiosis type, while the cultivars Pela Mão, Arroz do Governo, Cana Roxa, Come Cru Branco, Bico Preto, Pingo de Ouro, Matão, Gojobinho, Buriti, Rabo de Burro, Poupa Preguiça, Vermelho Trinca Ferro, Miúdo Branco, Ligeiro Curto, Vermelho Agulha, Agulhão, Marabá Branco, Gojoba $\backslash 75$ Dias $\backslash$ Ligeiro, Pingo D’ Água, Arroz Comum and Vermelho were revealed as probably tolerant to attack of the stem bug and therefore should be explored in breeding programs for resistance to T. limbativentris.

KEY WORDS: Oryza sativa L., plant breeding, resistance of plants to insects, resistance of types.

\section{INTRODUÇÃO}

O percevejo-do-colmo (Tibraca limbativentris Stal, 1860) éa praga de maior expressãoeconômica na cultura doarroz(Oryzasativa L.) no Brasil provocando prejuízos em termos de produtividade de até $90 \%$. Ataca os colmos das plantas com mais de 20 dias de idade sendo seus danos caracterizados pela morte parcial ou total da parte central dos colmos sintoma este conhecido por "coração morto" (FERREIRA et al., 1997).

A principal forma de controle do percevejo-docolmo tem sido a utilização do controle químico que vem de encontro com limitações de ordem econômica e ambiental, havendo a necessidade de se pesquisar e propor medidas alternativas de controle. As eventuais proposições deverão ser de baixo custo e de fácil adoção. Nesse sentido, a utilização de cultivares resistentes ao percevejo-do-colmo apresenta grande potencial para minimizar os danos causados por esse inseto.

Entre tais métodos, o emprego de cultivares resistentes é considerado ideal, uma vez que as populações das pragas podem ser mantidas abaixo de seus níveis de danos, reduzindo distúrbios ambientais e sem exigir conhecimentos específicos do agricultor, e nem custos adicionais (LARA, 1991). A resistência de plantas a insetos pode ser devida a três mecanismos: antixenose, tolerância e antibiose(PAINTER,1951).Uma planta apresenta a tolerância como mecanismo de resistência quando é menos danificada em relação a outras, sob um mesmo nível de infestação por uma determinada praga, sem afetar o comportamento ou a biologia da espécie fitófaga. Enquanto a antibiose caracteriza-se por proporcionar a redução da população de pragas, exercendo um efeito adverso ou mesmo letal sobre a biologia do inseto (LARA, 1991).

Há pouca informação sobre a resistência de cultivares de arroz à T. limbativentris a pesar da realização de alguns trabalhos a respeito (TRUILLO, 1970; FERREIRA et al., 1986; SOUSA; RODRIGUES, 1990). Ainda não foram desenvolvidos nem selecionados cultivares com essa característica para fins comerciais, e a seleção de arroz para resistência ao percevejo-do-colmo seria baseada principalmente em cultivares com alta capacidade de perfilhamento e associada a um menor número de perfilhos danificados (FERREIRAet al.,1997).

Neste trabalho objetivou-se avaliar a resistência de 64 cultivares de arroz ao ataque do percevejo-docolmo T. limbativentris a fim de indicar os mais promis- sores em futuros programas de melhoramento oferecendo novas ferramentas para o seu controle.

\section{MATERIAL E MÉTODOS}

O experimento foi conduzido em casa-de-vegetação no Centro Nacional de Pesquisa de Arroz e Feijão - CNPAF/EMBRAPA, no Município de Santo Antônio de Goiás, GO, no período de dezembro de 2004 a fevereiro de 2005. Foram utilizados 64 cultivares de arroz (Tabela 1), sendo 60 considerados tradicionais (FONSECA et al., 1982) e quatro outros cultivares mais atuais utilizados como testemunhas Cica 8, BR IRGA 409 (Ferreira etal., 1997), Bonança (Pereira et al.,2002) e Primavera (SouzA et al., 2006). As sementes dos 64 cultivares de arroz foram fornecidas pelo Banco Ativo de Germoplasma localizado no CNPAF/Embrapa.

Inicialmente, os 64 cultivares foram semeados (17/12/04) no solo do telado, em covas na densidade de 15 sementes, espaçados em 0,40 m, adotando-se o delineamento de blocos aumentados de Federer com dez repetições. A utilização deste tipo de delineamento deveu-se a pequena disponibilidade de sementes e por ser utilizado em etapas inicias de um programa de melhoramento de plantas quando o número de cultivares costuma ser alto conforme relatado por ZiMMERMANN (2004). Em cada bloco foram distribuídos seis dos 60 cultivares tradicionais e os quatro cultivares testemunhas. Foram utilizadas cinco ninfas de T. limbativentris com idade de, no máximo, 5 horas após a primeira ecdise provenientes de adultos alimentados em plantas da cultivar de arroz irrigado BR IRGA 409 e gaiolas cilindricas com base de PVC $(0,25$ $\mathrm{m}$ de diâmetro $\mathrm{x}$,20 $\mathrm{m}$ de altura) revestida por tecido fino ("voile").

Vinte dias após a semeadura, contou-se o número inicial de perfilhos e, em seguida, as plantas de cada cova foram isoladas com as gaiolas e infestadas com cinco ninfas (inicio do segundo instar). Ao final do experimento contou-se o número de colmos normais, danificados e os que foram emitidos após a infestação. Quando surgiram os primeiros insetos adultos nos cultivares testemunhas, cerca de 35 dias após a infestação, o experimento foi encerrado. As gaiolas foram removidas e os percevejos contados e coletados em saquinhos de papel para cada tratamento; os colmos com e sem "coração morto" foram contados. 
Os saquinhos contendo os percevejos foram colocados em congelador por 24 horas, depois em estufa a $50^{\circ} \mathrm{C}$ por 36 horas e a seguir pesados em balança de precisão.

Os caracteres avaliados foram: número de ninfas vivas (NNV), número de insetos adultos vivos (NAV), massa seca individual (MSI) e massa seca total (MST) de $T$. limbativentris expressas em $\mathrm{mg}$, colmos normais (CN), colmos emitidos após infestação (CEA) e colmos danificados (CD). A divisão da massa seca total pelo número de insetos vivos (ninfas + adultos) resultou na massa secaindividual dosinsetos. Onúmero decolmos normais foi obtido da subtração dos colmos finais pelo número de colmos com a sintomatologia "coração morto" visível adicionado aos colmos com "coração morto" invisível. O sintoma "coração morto" invisível foi detectado com o auxílio de um estilete no qual realizou-se um corteem todos os colmos das plantas de cada tratamento para visualizar ou não se a folha central encontrava-se morta dentro dele. Os colmos danificados foram obtidos por meio do somatório de colmos com "coração morto" visível e invisível.

Os caracteres foram analisados sem e com transformação em $\sqrt{X+0,5}$. Os que sofreram transformação foram: número de ninfas vivas (NNV), número de insetos adultos vivos (NAV), massa seca individual (MSI) e total (MST), colmos normais (CN) e colmos danificados (CD). Utilizou-se o programa computacional GENES (CRUZ, 2001) e as médias foram comparadas pelo teste de Scott \& Knott (SCOTT;KNOTT, 1974), no nível de $5 \%$ de probabilidade.

\section{RESULTADOS E DISCUSSÃO}

Foram observadas diferenças significativas em todos os caracteres avaliados (Tabela 1). Os cultivares Nenenzinho, Miúdo Branco, Lageado Ligeiro,
Guabirú, Branco Tardão, Agulhinha do Seco, Arroz do Governo, Arroz Misturado, Vermelho Trinca Ferro, Vermelhão, Chatão, Cutião, Vermelho, Bacaba Branco, Catetão, Buriti e Bacaba diferiram significativamente dos demais cultivares por apresentarem os menores índices de sobrevivência das ninfas (Tabela1). Oscultivares quecausaram menor sobrevivência sobre os insetosadultosforam Nenenzinho, Branco Tardão, Agulha, Arroz Comum, Vermelho, Pingo D'Água, Marabá Branco, Come Cru Vermelho e Agulhão que diferiram significativamente dos registrados nos cultivares Buriti, Miúdo Branco, Ligeiro Curto, C12\IAC 1246, Bacaba, Pingo de Ouro, Arroz Arara, Matão, Cana Roxa, Bico Preto, Rabo de Burro, Poupa Preguiça, Gojobinho, Zebu Ligeiro, Vermelho Trinca Ferro, Bacaba Branco, Come Cru Branco, Catetão e Tardão Vermelho que foram mais favoráveis à sobrevivência dos insetos adultos (Tabela 1).

Observou-se que os insetos criados sobre os cultivares Agulhão, Arroz Comum, Pingo D'Água e Marabá Branco apresentaram menor massa seca individual enquanto nos cultivares Arroz Comum, Agulhão, Pingo D’Água e Marabá Branco verificouse menor massa seca total (Tabela 1).

Esses resultados estão em conformidade com a situação em que indivíduos com menor peso, criados sobre plantas resistentes, evidenciam que os cultivares provavelmente possuem características da antibiose, aliada à mortalidade de formas jovens e de insetos adultos (LARA, 1991).

A resistência do tipo antibiose do acesso $\mathrm{CNPH}$ 101 de Lycopersicon peruvianum à traça do tomateiro Tuta absoluta (Meyrick) (Lepidoptera: Gelechiidae) foi estudada por SuINAGA et al. (2004). Os autores verificaram que o acesso CNPH 101 deL.peruvianum apresentou resistência do tipo antibiose constatada pela mortalidade larval e duração da fase pupal dos insetos.

Tabela 1 - Comparação entre médias de 64 cultivares de Oryza sativa em relação a sete caracteres de resistência a Tibraca limbativentris. Santo Antônio de Goiás, GO, 2005.

\begin{tabular}{llllrrrr}
\hline & \multicolumn{5}{c}{ Caracteres de resistência } \\
\cline { 2 - 7 } Cultivares & $\mathrm{NNV}^{1 * *}$ & $\mathrm{NAV}^{* *}$ & MSI $^{* *}$ & MST $^{* *}$ & $\mathrm{CEA}$ & $\mathrm{CN}^{* *}$ & $\mathrm{CD}^{* *}$ \\
\hline 1) Arroz Misturado & $0,63 \mathrm{~d}$ & $1,78 \mathrm{~b}$ & $7,65 \mathrm{~b}$ & $11,19 \mathrm{~b}$ & $16,25 \mathrm{~b}$ & $4,56 \mathrm{~b}$ & $2,49 \mathrm{c}$ \\
2) Agulha & $1,57 \mathrm{a}$ & $0,69 \mathrm{~d}$ & $3,93 \mathrm{c}$ & $6,67 \mathrm{c}$ & $3,25 \mathrm{c}$ & $2,94 \mathrm{~d}$ & $3,13 \mathrm{c}$ \\
3) Arroz Asa & $0,80 \mathrm{c}$ & $1,26 \mathrm{c}$ & $7,32 \mathrm{~b}$ & $7,94 \mathrm{c}$ & $7,50 \mathrm{c}$ & $3,11 \mathrm{~d}$ & $3,74 \mathrm{~b}$ \\
4) Arroz Pubero & $1,47 \mathrm{a}$ & $1,71 \mathrm{~b}$ & $6,40 \mathrm{~b}$ & $11,65 \mathrm{~b}$ & $8,50 \mathrm{c}$ & $3,32 \mathrm{c}$ & $3,60 \mathrm{~b}$ \\
5) Amarelão & $0,89 \mathrm{c}$ & $0,91 \mathrm{c}$ & $5,38 \mathrm{c}$ & $6,12 \mathrm{c}$ & $8,25 \mathrm{c}$ & $3,27 \mathrm{c}$ & $3,19 \mathrm{c}$ \\
6) Arroz do Governo & $0,63 \mathrm{~d}$ & $1,78 \mathrm{~b}$ & $8,04 \mathrm{a}$ & $11,74 \mathrm{~b}$ & $19,25 \mathrm{~b}$ & $6,47 \mathrm{a}$ & $1,92 \mathrm{c}$ \\
7) Agulhão & $1,50 \mathrm{a}$ & $0,18 \mathrm{~d}$ & $0,78 \mathrm{e}$ & $0,84 \mathrm{e}$ & $0,10 \mathrm{e}$ & $3,07 \mathrm{~d}$ & $1,55 \mathrm{~d}$ \\
8) Arroz Comum & $0,92 \mathrm{c}$ & $0,56 \mathrm{~d}$ & $0,74 \mathrm{e}$ & $0,93 \mathrm{e}$ & $9,75 \mathrm{c}$ & $5,26 \mathrm{~b}$ & $0,80 \mathrm{~d}$ \\
9) Agulhinha do Seco & $0,63 \mathrm{~d}$ & $1,78 \mathrm{~b}$ & $4,75 \mathrm{c}$ & $7,06 \mathrm{c}$ & $6,25 \mathrm{c}$ & $2,77 \mathrm{~d}$ & $3,75 \mathrm{~b}$
\end{tabular}

Continua... 
Tabela 1 - Cotinuação.

\begin{tabular}{|c|c|c|c|c|c|c|c|}
\hline \multirow[b]{2}{*}{ Cultivares } & \multicolumn{7}{|c|}{ Caracteres de resistência } \\
\hline & $\mathrm{NNV}^{1 * *}$ & $\mathrm{NAV}^{* *}$ & $\mathrm{MSI}^{* *}$ & $\mathrm{MST}^{* *}$ & CEA & $\mathrm{CN}^{* *}$ & $C D^{* *}$ \\
\hline 10) Arroz Arara & $0,96 c$ & $2,33 a$ & $5,90 \mathrm{~b}$ & $14,87 \mathrm{~b}$ & $18,50 \mathrm{~b}$ & $2,82 d$ & $4,91 \mathrm{a}$ \\
\hline 11) Arroz Projeto & $1,83 a$ & $0,84 \mathrm{c}$ & $4,20 \mathrm{c}$ & $6,64 c$ & $7,50 \mathrm{c}$ & $3,32 \mathrm{c}$ & $3,75 b$ \\
\hline 12) Branco & $1,47 \mathrm{a}$ & $2,00 \mathrm{~b}$ & $8,20 a$ & $16,85 a$ & $13,50 \mathrm{~b}$ & $2,70 \mathrm{~d}$ & $4,89 \mathrm{a}$ \\
\hline 13) Bacaba & $0,29 \mathrm{~d}$ & $2,42 \mathrm{a}$ & $9,93 a$ & $16,29 a$ & $17,50 \mathrm{~b}$ & $1,90 \mathrm{e}$ & $6,02 a$ \\
\hline 14) Branco Tardão & $0,63 d$ & $0,70 \mathrm{~d}$ & $5,60 c$ & $7,09 c$ & $4,50 \mathrm{c}$ & $4,60 \mathrm{~b}$ & $2,12 \mathrm{c}$ \\
\hline 15) Bacaba Branco & $0,41 \mathrm{~d}$ & $2,11 a$ & $6,73 b$ & $13,30 b$ & $14,25 b$ & $4,55 b$ & $3,43 b$ \\
\hline 16) Bico Ganga & $1,05 b$ & $1,59 b$ & $8,30 \mathrm{a}$ & $13,91 b$ & $9,75 c$ & $4,60 \mathrm{~b}$ & $2,74 \mathrm{c}$ \\
\hline 17) Bico Preto & $0,92 \mathrm{c}$ & $2,19 a$ & $8,27 \mathrm{a}$ & $17,94 a$ & $28,75 a$ & $3,76 c$ & $5,70 \mathrm{a}$ \\
\hline 18) Buriti & $0,38 \mathrm{~d}$ & $2,55 a$ & $7,21 b$ & $16,40 \mathrm{a}$ & $26,25 a$ & $4,47 \mathrm{~b}$ & $4,64 a$ \\
\hline 19) C-2 & $0,96 c$ & $1,36 \mathrm{c}$ & $5,38 \mathrm{c}$ & $7,58 \mathrm{c}$ & $6,50 c$ & $3,58 \mathrm{c}$ & $2,84 \mathrm{c}$ \\
\hline 20) Cutião Vermelho & $0,41 \mathrm{~d}$ & $1,86 b$ & $7,72 b$ & $13,29 b$ & $12,25 c$ & $4,66 b$ & $2,24 c$ \\
\hline 21) Catetão & $0,38 \mathrm{~d}$ & $2,07 a$ & $7,08 b$ & $12,72 b$ & $20,25 b$ & $5,27 b$ & $3,50 \mathrm{~b}$ \\
\hline 22) C 12/IAC 1246 & $0,80 \mathrm{c}$ & $2,42 \mathrm{a}$ & $7,07 \mathrm{~b}$ & $12,65 b$ & $16,50 \mathrm{~b}$ & $4,77 \mathrm{~b}$ & $2,62 c$ \\
\hline 23) Cana Roxa & $0,92 \mathrm{c}$ & $2,19 a$ & $7,32 b$ & $15,82 \mathrm{a}$ & $29,75 a$ & $6,22 a$ & $3,76 b$ \\
\hline 24) Come Cru Branco & $1,25 b$ & $2,07 a$ & $5,93 b$ & $13,51 b$ & $30,25 a$ & $5,68 \mathrm{a}$ & $3,92 b$ \\
\hline 25) Come Cru Vermelho & $1,14 \mathrm{~b}$ & $0,18 \mathrm{~d}$ & $2,56 \mathrm{~d}$ & $3,52 d$ & $0,11 \mathrm{e}$ & $2,80 \mathrm{~d}$ & $1,94 \mathrm{c}$ \\
\hline 26) Cutião & $0,83 c$ & $1,29 \mathrm{c}$ & $6,93 b$ & $8,44 \mathrm{c}$ & $8,75 c$ & $3,86 \mathrm{c}$ & $3,21 c$ \\
\hline 27) Chatão & $0,41 \mathrm{~d}$ & $1,57 \mathrm{~b}$ & $6,82 b$ & $9,63 c$ & $17,25 b$ & $5,26 b$ & $2,44 \mathrm{c}$ \\
\hline 28) Chatão Vermelho & $0,96 \mathrm{c}$ & $1,65 b$ & $5,28 c$ & $9,79 \mathrm{c}$ & $17,50 \mathrm{~b}$ & $4,98 b$ & $2,50 \mathrm{c}$ \\
\hline 29) Douradão & $0,92 \mathrm{c}$ & $1,07 \mathrm{c}$ & $9,46 a$ & $9,58 c$ & $9,75 c$ & $4,58 b$ & $2,21 c$ \\
\hline 30) Desconhecido Branco & $0,96 c$ & $1,00 \mathrm{c}$ & $6,97 b$ & $7,06 \mathrm{c}$ & $1,50 \mathrm{~d}$ & $3,46 \mathrm{c}$ & $2,11 c$ \\
\hline 31) Gojobinho & $0,83 \mathrm{c}$ & $2,19 a$ & $10,34 a$ & $20,96 a$ & $26,75 a$ & $4,75 b$ & $5,03 a$ \\
\hline 32) Gojoba/75 Dias/Ligeiro & $0,80 \mathrm{c}$ & $1,26 \mathrm{c}$ & $6,77 b$ & $7,39 c$ & $7,50 \mathrm{c}$ & $4,45 b$ & $1,21 d$ \\
\hline 33) Guabirú & $0,63 d$ & $1,05 c$ & $3,88 \mathrm{c}$ & $3,98 \mathrm{~d}$ & $0,50 \mathrm{~d}$ & $3,44 \mathrm{c}$ & $3,14 \mathrm{c}$ \\
\hline 34) Jatobá & $1,25 b$ & $1,78 \mathrm{~b}$ & $6,39 b$ & $13,12 b$ & $15,25 b$ & $5,27 \mathrm{~b}$ & $2,46 c$ \\
\hline 35) Lageado & $1,05 b$ & $1,84 \mathrm{~b}$ & $4,96 c$ & $9,65 c$ & $7,75 c$ & $3,09 \mathrm{~d}$ & $4,93 a$ \\
\hline 36) Ligeiro & $1,50 \mathrm{a}$ & $0,90 \mathrm{c}$ & $7,21 b$ & $10,55 c$ & $18,25 b$ & $4,88 \mathrm{~b}$ & $2,65 c$ \\
\hline 37) Lageado Ligeiro & $0,63 d$ & $1,82 b$ & $3,53 c$ & $7,82 \mathrm{c}$ & $0,20 \mathrm{e}$ & $2,00 \mathrm{e}$ & $3,38 b$ \\
\hline 38) Ligeiro Curto & $0,80 \mathrm{c}$ & $2,42 \mathrm{a}$ & $8,76 a$ & $16,05 a$ & $21,50 a$ & $2,77 d$ & $5,55 a$ \\
\hline 39) Matão & $0,89 \mathrm{c}$ & $2,32 \mathrm{a}$ & $7,45 b$ & $16,92 \mathrm{a}$ & $27,25 a$ & $5,17 \mathrm{~b}$ & $4,17 \mathrm{~b}$ \\
\hline 40) Marabá & $0,80 \mathrm{c}$ & $1,78 \mathrm{~b}$ & $5,22 \mathrm{c}$ & $7,10 \mathrm{c}$ & $14,50 \mathrm{~b}$ & $4,21 b$ & $3,58 b$ \\
\hline 41) Marabá Branco & $1,05 b$ & $0,43 \mathrm{~d}$ & $0,58 \mathrm{e}$ & $0,65 e$ & $0,40 \mathrm{~d}$ & $3,49 \mathrm{c}$ & $1,41 d$ \\
\hline 42) Miúdo Branco & $0,63 \mathrm{~d}$ & $2,54 a$ & $9,06 a$ & $20,29 a$ & $22,25 \mathrm{a}$ & $3,57 \mathrm{c}$ & $5,09 a$ \\
\hline 43) Mato Grosso & $1,47 \mathrm{a}$ & $0,84 \mathrm{c}$ & $4,35 c$ & $5,28 \mathrm{c}$ & $2,50 \mathrm{~d}$ & $3,02 d$ & $2,71 c$ \\
\hline 44) Mucuim/Miúdo & $0,96 c$ & $2,00 \mathrm{~b}$ & $9,52 a$ & $17,07 \mathrm{a}$ & $12,50 \mathrm{c}$ & $3,72 \mathrm{c}$ & $4,54 a$ \\
\hline 45) Nenenzinho & $0,63 d$ & $0,70 \mathrm{~d}$ & $5,36 c$ & $6,12 c$ & $0,50 \mathrm{~d}$ & $4,21 b$ & $2,12 c$ \\
\hline 46) Palha Murcha/Cuchilão & $0,92 \mathrm{c}$ & $1,43 \mathrm{c}$ & $6,51 b$ & $8,95 c$ & $13,75 b$ & $3,26 c$ & $4,39 \mathrm{~b}$ \\
\hline 47) Pela Mão & $1,34 \mathrm{a}$ & $1,94 b$ & $8,27 a$ & $16,81 \mathrm{a}$ & $28,75 a$ & $6,55 a$ & $3,68 b$ \\
\hline 48) Pingo de Ouro & $0,83 \mathrm{c}$ & $2,41 \mathrm{a}$ & $7,98 \mathrm{a}$ & $17,81 \mathrm{a}$ & $27,75 a$ & $4,02 \mathrm{c}$ & $5,14 \mathrm{a}$ \\
\hline 49) Poupa Preguiça & $0,83 c$ & $2,19 a$ & $9,23 a$ & $18,73 a$ & $22,75 a$ & $5,36 b$ & $4,23 b$ \\
\hline 50) Pingo D'Água & $1,56 a$ & $0,43 \mathrm{~d}$ & $0,58 \mathrm{e}$ & $0,65 \mathrm{e}$ & $0,75 d$ & $3,85 c$ & $0,90 \mathrm{~d}$ \\
\hline 51) Rabo de Burro & $0,83 c$ & $2,19 a$ & $8,69 a$ & $17,66 a$ & $22,75 a$ & $3,29 c$ & $5,44 \mathrm{a}$ \\
\hline 52) Saia Velha & $0,92 \mathrm{c}$ & $1,07 \mathrm{c}$ & $8,06 a$ & $8,35 c$ & $15,75 b$ & $5,05 b$ & $2,83 c$ \\
\hline 53) Tardão Vermelho & $1,14 \mathrm{~b}$ & $2,07 a$ & $7,00 \mathrm{~b}$ & $14,11 b$ & $7,25 c$ & $2,58 \mathrm{~d}$ & $3,63 b$ \\
\hline 54) Taboca & $1,47 \mathrm{a}$ & $0,84 \mathrm{c}$ & $2,74 \mathrm{~d}$ & $3,67 d$ & $2,50 \mathrm{~d}$ & $3,59 \mathrm{c}$ & $2,23 c$ \\
\hline 55) Vermelho & $1,47 \mathrm{a}$ & $0,48 \mathrm{~d}$ & $2,73 \mathrm{~d}$ & $3,53 \mathrm{~d}$ & $5,50 \mathrm{c}$ & $4,52 b$ & $0,47 \mathrm{~d}$ \\
\hline 56) Vermelhão & $0,41 \mathrm{~d}$ & $1,86 b$ & $7,23 b$ & $12,43 b$ & $14,25 b$ & $4,08 \mathrm{c}$ & $3,95 b$ \\
\hline 57) Vermelhinho/75 Dias/Ligeiro & $1,05 b$ & $1,84 \mathrm{~b}$ & $5,18 \mathrm{c}$ & $10,10 \mathrm{c}$ & $11,75 c$ & $1,90 \mathrm{e}$ & $5,53 a$ \\
\hline 58) Vermelho Agulha & $1,05 b$ & $0,95 c$ & $7,18 b$ & $7,40 \mathrm{c}$ & $6,75 c$ & $4,40 \mathrm{~b}$ & $1,77 d$ \\
\hline 59) Vermelho Trinca Ferro & $0,41 \mathrm{~d}$ & $2,11 a$ & $8,05 a$ & $15,94 a$ & $22,25 \mathrm{a}$ & $3,96 \mathrm{c}$ & $4,42 b$ \\
\hline
\end{tabular}

Continua... 
Tabela 1 - Cotinuação.

\begin{tabular}{llllrrrr}
\hline & \multicolumn{5}{c}{ Caracteres de resistência } \\
\cline { 2 - 7 } Cultivares & $\mathrm{NNV}^{1 * *}$ & $\mathrm{NAV}^{* *}$ & MSI $^{* *}$ & MST $^{* *}$ & $\mathrm{CEA}$ & $\mathrm{CN}^{* *}$ & $\mathrm{CD}^{* *}$ \\
\hline 60) Zebu Ligeiro & $0,96 \mathrm{c}$ & $2,12 \mathrm{a}$ & $5,25 \mathrm{c}$ & $13,43 \mathrm{~b}$ & $17,50 \mathrm{~b}$ & $4,23 \mathrm{~b}$ & $4,06 \mathrm{~b}$ \\
61) Cica 8* & $1,11 \mathrm{~b}$ & $1,63 \mathrm{~b}$ & $5,45 \mathrm{c}$ & $10,29 \mathrm{c}$ & $14,00 \mathrm{~b}$ & $3,87 \mathrm{c}$ & $3,99 \mathrm{~b}$ \\
62) Bonança* & $1,16 \mathrm{~b}$ & $0,98 \mathrm{c}$ & $3,26 \mathrm{c}$ & $4,81 \mathrm{c}$ & $6,30 \mathrm{c}$ & $4,09 \mathrm{c}$ & $2,75 \mathrm{c}$ \\
63) BR IRGA 409* & $1,34 \mathrm{a}$ & $1,10 \mathrm{c}$ & $4,73 \mathrm{c}$ & $7,07 \mathrm{c}$ & $11,50 \mathrm{c}$ & $3,92 \mathrm{c}$ & $3,65 \mathrm{~b}$ \\
64) Primavera* & $1,08 \mathrm{~b}$ & $1,32 \mathrm{c}$ & $5,57 \mathrm{c}$ & $8,40 \mathrm{c}$ & $11,20 \mathrm{c}$ & $3,95 \mathrm{c}$ & $3,49 \mathrm{~b}$ \\
\hline
\end{tabular}

${ }^{1} \mathrm{NNV}=$ número de ninfas vivas, $\mathrm{NAV}=$ número de insetos adultos vivos, $\mathrm{MSI}=$ massa seca individual e MST = massa seca total de $T$. limbativentris por $\mathrm{mg}, \mathrm{CEA}=$ colmos emitidos após infestação, $\mathrm{CN}=$ colmos normais e $\mathrm{CD}=$ colmos danificados.

* Testemunhas; Médias seguidas de letras distintas na coluna diferem significativamente entre si, pelo teste de Scott \& Knott, a $5 \%$ de probabilidade.

**Dados transformados = número de ninfas vivas (NNV), número de insetos adultos vivos (NAV), massa seca individual (MSI) e total (MST), colmos normais (CN) e colmos danificados (CD).

Quanto ao número de colmos normais constatou-se que os cultivares Pela Mão, Arroz do Governo, Cana Roxa e Come Cru Branco foram os que mais apresentaram colmos sadios ao término do experimento diferindo significativamente dos cultivares Lageado Ligeiro, Vermelhinho $\backslash 75$ Dias $\backslash$ Ligeiro e Bacaba que apresentaram menor número (Tabela 1). Os cultivares que mais perfilharam após infestação foram Come Cru Branco, Cana Roxa, Pela Mão, Bico Preto, Pingo de Ouro, Matão, Gojobinho, Buriti, Rabo de Burro, Poupa Preguiça, Vermelho Trinca Ferro, Miúdo Branco e Ligeiro Curto diferindo significativamente dos cultivares Lageado Ligeiro, Come Cru Vermelho e Agulhão que perfilharam menos.

O número de colmos danificados observados nos cultivares Bacaba, Bico Preto, Ligeiro Curto, Vermelhinho $\backslash 75$ Dias $\backslash$ Ligeiro, Rabo de Burro, Pingo de Ouro, Miúdo Branco, Gojobinho, Lageado, Arroz Arara, Branco, Buriti e Mucuim \Miúdo foi maior quando comparado com os cultivares Vermelho Agulha, Agulhão, Marabá Branco, Gojoba $\backslash 75$ Dias $\backslash$ Ligeiro, Pingo D'Água, Arroz Comum e Vermelho que apresentaram as menores médias.

Dessa forma pode-se inferir que o comportamento dos cultivares frente ao ataque do $T$. limbativentris revela que, possivelmente, eles são portadores de resistência do tipo tolerância e que estão de acordo com FERreiraet al. (1986) que estudaram, em dois experimentos, a possibilidade de identificar fontes de resistência de arroz ao percevejo-do-colmo. Os autores observaram que é preferível selecionar cultivares e linhagens altamente perfilhadoras e com uma quantidade de colmos danificados a mais baixa possível.

\section{CONCLUSÃO}

De maneira geral, pode-se inferir que os cultivares Nenenzinho, Miúdo Branco, Lageado Ligeiro, Guabirú, Branco Tardão, Agulhinha do Seco, Arroz do Governo, Arroz Misturado, Vermelho Trinca Ferro, Vermelhão, Chatão, Cutião, Vermelho, Bacaba Branco, Catetão, Buriti, Bacaba, Agulha, Arroz Comum, Vermelho, Pingo D'Água, Marabá Branco, Come Cru Vermelho e Agulhão destacaram-se como resistentes possivelmente do tipo antibiose, enquanto os cultivares Pela Mão, Arroz do Governo, Cana Roxa, Come Cru Branco, Bico Preto, Pingo de Ouro, Matão, Gojobinho, Buriti, Rabo de Burro, Poupa Preguiça, Vermelho Trinca Ferro, Miúdo Branco, Ligeiro Curto, Vermelho Agulha, Agulhão, Marabá Branco, Gojoba $\backslash 75$ Dias $\backslash$ Ligeiro, Pingo D' Água, Arroz Comum e Vermelho provavelmente são tolerantes ao ataque do percevejo-do-colmo devendo, portanto, ser devidamente explorados em programas de melhoramento para resistência a T. limbativentris.

\section{AGRADECIMENTOS}

Ao Centro Nacional de Pesquisa de Arroz e Feijão/Embrapa, Universidade Estadual do Maranhão/ UEMA e ao ilustre Dr. Evane Ferreira (in memoriam) que com compreensão e humildade contribuiu para a conclusão desta pesquisa.

\section{REFERÊNCIAS}

CRUZ, C. D. Programa GENES - versão Windows.

Aplicativo computacional em genética e estatística. Viçosa: UFV, 2001. p.648. 
FERREIRA, E.; MARTINS, J. F. S.; RANGEL, P. H. N.; CUTRIM, V. A. Resistência de arroz ao percevejo do colmo. Pesquisa Agropecuária Brasileira, v.21, n.5, p.565$569,1986$.

FERREIRA, E.; ZIMMERMANN, F.J.D.; SANTOS, A.B.; NEVES, B.P. O percevejo do colmo na cultura do arroz. Goiânia: EMBRAPA-CNPAF, 1997, 43p. (Documentos, 75).

FONSECA, J. R.; RANGEL, P. H.; BEDENDO, I. P.; SILVEIRA, P. M.; GUIMARÃES, E. P.; CORANDIN, L. Características botânicas e agronômicas de cultivares e raças regionais de arroz (Oryza sativa L.) coletadas no Estado do Maranhão. Santo Antônio de Goiás: Embrapa Arroz e Feijão, 1982. 42p. (Boletim de Pesquisa, 1).

LARA, F.M. Princípios de resistência de plantas aos insetos. 2.ed. São Paulo: Ícone, 1991. p.336.

PAINTER, R.H. Insect resistance in crop plants. New York: Macmillan, 1951. 520p.

PEREIRA, B.G.; BARRIGOSSI, J.A.F.; FERREIRA, E. Relação injúria e dano do percevejo-do-colmo do arroz, Tibraca limbativentris (Hemiptero: Pentatomidae). In: CONGRESSO DA CADEIA PRODUTIVA DE ARROZ, 1. REUNIÃO NACIONAL DE PESQUISA DE ARROZ 7., REUNIÃO NACIONAL DE PESQUISA DE ARROZ RENAPA, 2002, Florianópolis, SC. Anais. Santo Antônio de Goiás: Embrapa Arroz e Feijão, 2002. p.506-509.

SCOTT, A J.; KNOTT, M.A. A cluster analysis method for grouping means in the analysis of variance. Biometrics, v.30, n.3, p.507-512, 1974.
SOUZA, I.S.; RODRIGUES, F.J. O. Estudo da resistência varietal ao percevejo Tibraca limbativentris em arroz de sequeiro. In: REUNIÃO NACIONAL DE PESQUISA DE ARROZ, 1990, Goiânia, GO. Resumos. Goiânia, GO, 1990. p.33.

SOUZA, J.R.; FERREIRA, E.; CHAGAS, E.F. Avaliação de variedades de arroz cultivadas no Estado do Maranhão para antibiose a ninfas de Tibraca limbativentris (Stal, 1860) (Hemiptero: Pentatomidae). In: CONGRESSO BRASILEIRO DA CADEIA PRODUTIVA DE ARROZ, 2., REUNIÃO NACIONAL DE PESQUISA DE ARROZ, 8., 2006, Brasília, DF. Anais. Santo Antônio de Goiás: Embrapa Arroz e Feijão, 2006.

SUINAGA, F.A.; PICANÇO, M.C.; MOREIRA, M.D.; SEMEÃO, A.A.; MAGALHÃES, S.T.V. Resistência por antibiose de Lycopersicon peruvianum à traça do tomateiro. Horticultura Brasileira, v.22, n.2, p.281-285, 2004.

TRUJILLO, M.R. Contribuição ao conhecimento do dano e biologia de Tibraca limbativentris Stal, 1860 (Hemiptero: Pentatomidae) praga da cultura do arroz. 1970. 63f. Dissertação (Mestrado em Agronomia - Entomologia) Escola Superior de Agricultura Luiz de Queiroz, Piracicaba, 1970.

ZIMMERMANN, F.J.P. Estatística aplicada á pesquisa agrícola. Santo Antônio de Goiás: Embrapa Arroz e Feijão, 2004. 402p.

Recebido em 28/11/07

Aceito em 10/9/08 